\title{
Reversals of chance in paradoxical games
}

\author{
P. Amengual ${ }^{\mathrm{a}, *}$, P. Meurs ${ }^{\mathrm{b}}$, B. Cleuren ${ }^{\mathrm{b}}$, R. Toral ${ }^{\mathrm{a}}$ \\ ${ }^{a}$ Departament de Física and Institut Mediterrani d'Estudis Avançats, Universitat de les Illes Balears, E07122 Palma de Mallorca, Spain \\ ${ }^{\mathrm{b}}$ Hasselt University, B-3590 Diepenbeek, Belgium
}

Received 12 January 2006; received in revised form 9 March 2006

Available online 24 April 2006

\begin{abstract}
We present two collective games with new paradoxical features when they are combined. Besides reproducing the socalled Parrondo effect, where a winning game is obtained from the alternation of two fair games, there also exists a current inversion when varying the mixing probability between the games. We show that this is a new effect insofar one of the games is an unbiased random walk without internal structure. We present a detailed study by means of a discrete-time Markov chain analysis, obtaining analytical expressions for the stationary probabilities for a finite number of players. We also provide qualitative insight into this current inversion effect.
\end{abstract}

(C) 2006 Elsevier B.V. All rights reserved.

Keywords: Markov chain theory; Parrondo's paradox; Brownian ratchet

\section{Introduction}

In the past few years there has been an increasing interest in what is known in the literature as Parrondo's paradox [1-3]. This phenomenon shows that the alternation of two fair (or even losing) games can result in a winning game. These so-called Parrondo games were originally defined as follows: game $A$ is a simple coin tossing game, where the player wins or loses one unit of capital with probabilities $p^{A}$ and $1-p^{A}$, respectively. For game $B$ the winning probability depends on the capital of the player modulo three, governed by the set of probabilities $\left\{p_{B}^{1}, p_{B}^{2}, p_{B}^{3}\right\}$. In the (stochastic) combination of these games, either game $A$ or $B$ is played, with probabilities $\gamma$ and $1-\gamma$, respectively. The games are said to be fair/losing/winning when on average the player's capital stabilizes/decreases/increases.

In the first works about Parrondo games, to which we will further refer as the "original" games $A$ and $B$, the following parameter values were used: $p^{A}=\frac{1}{2}-\varepsilon, p_{B}^{1}=\frac{1}{10}-\varepsilon, p_{B}^{2}=p_{B}^{3}=\frac{3}{4}-\varepsilon$. When $\varepsilon=0$ both games $A$ and $B$ played separately are fair, whereas if $\varepsilon>0$ both games turn out to be losing. The Parrondo effect appears when the stochastic $(0<\gamma<1)$ or periodic combination of these fair/losing games results in a winning game.

These games were first devised in 1996 by the Spanish physicist Juan M.R. Parrondo, who presented them in unpublished form in Torino, Italy [4]. They served as a pedagogical illustration of the flashing ratchet [5], where directed motion is obtained from the random or periodic alternation of two relaxation potentials acting

\footnotetext{
*Corresponding author.

E-mail addresses: pau@imedea.uib.es (P. Amengual), pascal.meurs@uhasselt.be (P. Meurs).

$U R L:$ http://www.imedea.uib.es/ pau.
} 
on a Brownian particle, none of which individually produces any net flux. Only recently a quantitative relation has been established between the Brownian ratchet and Parrondo's games [6,7].

Cooperative versions of the games, played by a set of $N>1$ players, have also been studied. In Ref. [8], a set of $N$ players are arranged in a ring and each round a player is chosen randomly to play either game $A$ or $B$. The original game $A$ is combined with a new game $B$, for which the winning probability depends on the state (winner/loser) of the nearest neighbors of the selected player. A player is said to be a winner (loser) if he has won (lost) his last game. In Ref. [9] again a set of $N$ players is considered, but for this case game $A$ is replaced by a redistribution process where a player is chosen randomly to give away one coin of his capital to another player. When combining this new game with the original game $B$, the paradox is reproduced.

In this work we present a new version of collective games, where besides obtaining the desired result of a winning game out of two fair games, another feature appears: the games show under certain circumstances a current inversion when varying $\gamma$, i.e., the value of the mixing probability $\gamma$ determines whether you end up with a winning or a losing game $A+B$. As shown in Ref. [10], it is not possible to obtain a current inversion in a single player set-up using the standard rules of the original games when game $A$ is state independent. For the collective games considered here, we are able to obtain a current inversion even if one of the games used (game A) uses no information at all about the present state of the system. And so this current inversion is a genuine collective effect, without a corresponding analog in the single player game.

The paper is organized as follows: in Section 2 we present the games in detail as well as a theoretical analysis; in Section 3 we offer a qualitative picture of the impossibility of a current inversion using the original games; finally in Section 4 we draw the conclusions.

\section{The games}

The games will be played by a set of $N$ players. In each round, a player is selected randomly for playing. Then, with probabilities $\gamma$ and $1-\gamma$, respectively, game $A$ or $B$ is played. Game $A$ is the original game in which the selected player wins or loses one coin with probability $p^{A}$ and $1-p^{A}$, respectively. The winning probabilities in game $B$ depend on the collective state of all players. Again, as in Ref. [8], a player is said to be a winner or a loser when he has won or lost, respectively, his last game. More precisely, the winning probability can have three possible values, determined by the actual number of winners $i$ within the total number of players $N$, in the following way

$$
p_{i}^{B} \equiv \text { probability to win in game } B= \begin{cases}p_{B}^{1} & \text { if } i>\left\lceil\frac{2 N}{3}\right\rceil, \\ p_{B}^{2} & \text { if }\left\lceil\frac{N}{3}\right\rceil \leqslant i \leqslant\left\lceil\frac{2 N}{3}\right\rceil, \\ p_{B}^{3} & \text { if } i<\left\lceil\frac{N}{3}\right\rceil,\end{cases}
$$

where the brackets $\lceil x\rceil$ denote the nearest integer to the number $x$.

\subsection{Analysis of the games}

The main quantity of interest is the average gain of the collection of $N$ players when playing the stochastic game $A+B$. Since the winning probability of game $B$ only depends on the total number of winners, it suffices to describe the games using a set of $N+1$ different states $\left\{\sigma_{0}, \sigma_{1}, \ldots, \sigma_{N}\right\}$. The state $\sigma_{i}$ is the configuration where $i$ players are labelled as "winner" and $N-i$ as "loser". Transitions between the states will be determined by the forward transition probability $p_{i}$, the backward transition probability $q_{i}$, and the probability for remaining in the same state $r_{i}$, see Fig. 1.

Denoting as $P_{i}(t)$ the probability of finding the system in state $\sigma_{i}$ at the $t$ th round played, we can write the equation governing its time evolution as

$$
P_{i}(t+1)=p_{i-1} P_{i-1}(t)+r_{i} P_{i}(t)+q_{i+1} P_{i+1}(t),
$$




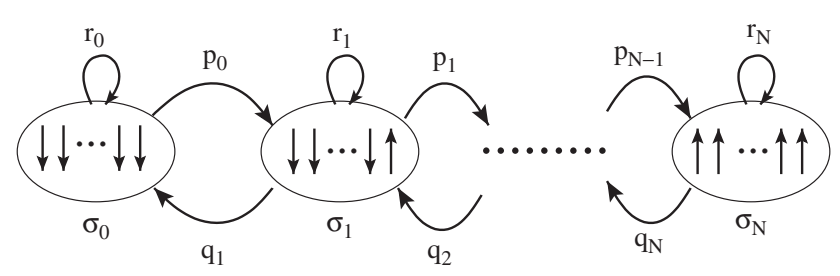

Fig. 1. Different states and allowed transitions for $N$ players. The arrows indicate the state of each player being a winner (arrow up) or a loser (arrow down).

with $0 \leqslant i \leqslant N$ and where the transition probabilities are given by

$$
\begin{aligned}
p_{i} & =\frac{N-i}{N}\left[\gamma p^{A}+(1-\gamma) p_{i}^{B}\right], \\
r_{i} & =\frac{2 i-N}{N}\left[\gamma p^{A}+(1-\gamma) p_{i}^{B}\right]+\frac{N-i}{N}, \\
q_{i} & =\frac{i}{N}\left[\gamma\left(1-p^{A}\right)+(1-\gamma)\left(1-p_{i}^{B}\right)\right] .
\end{aligned}
$$

These transition probabilities have been obtained through the following reasoning: if we recall that in state $i$ there are $N-i$ losers and $i$ winners, the only way that we can go forward to state $i+1$ is by choosing a player labelled as a loser - with probability $(N-i) / N$ - and that player winning the game. So if there is a probability $\gamma$ of playing game $A$ and a probability $1-\gamma$ of playing game $B$, the combined winning probability will be given by $\gamma p^{A}+(1-\gamma) p_{i}^{B}$. Considering these two contributions, the forward transition (3) from state $i$ to state $i+1$ is obtained. The transition probabilities $r_{i}$ and $q_{i}$ follow from the same reasoning.

The set of transition probabilities $\left(p_{i}, q_{i}, r_{i}\right)$ must satisfy the normalization condition $p_{i}+r_{i}+q_{i}=1$, which implies for the probabilities $P_{i}(t)$ that $\sum_{i=0}^{N} P_{i}(t)=1$, as long as $\sum_{i=0}^{N} P_{i}(t=0)=1$.

This system of $N+1$ equations can be solved in the stationary state, where the probabilities no longer depend on time $P_{i}(t)=P_{i}^{s t}$, and the general solution reads

$$
P_{i}^{s t}=\frac{1}{Z} p_{0} p_{1} \cdots p_{i-1} q_{i+1} q_{i+2} \cdots q_{N}
$$

where $0 \leqslant i \leqslant N$ and $Z$ is the normalization factor. Once the stationary probabilities are calculated, we can obtain the average winning probability over all states for the stochastic combination $A+B$ (mixing probability $\gamma$ ) from

$$
p_{w i n}^{A+B}=\sum_{i=0}^{N}\left[\gamma p^{A}+(1-\gamma) p_{i}^{B}\right] P_{i}^{s t} .
$$

The average gain can then easily be evaluated through the expression $J^{A+B}=2 p_{w i n}^{A+B}-1$. The properties of the separate games $A$ and $B$ can be obtained by replacing in the previous expressions $\gamma$ by 1 or 0 , respectively.

\subsubsection{The Parrondo effect}

The Parrondo effect appears when from the combination of two fair games, we obtain a winning game. Clearly, game $A$ is fair for $p^{A}=\frac{1}{2}$. For game $B$ the set of values $\left\{p_{B}^{1}, p_{B}^{2}, p_{B}^{3}\right\}$ giving a fair game is more difficult to determine because it depends on the total number of players $N$. The conditions on $p_{B}^{2}$ for a fair game $B$ have been found analytically by a symbolic manipulation program up to $N<13$. In Table 1 we find listed the conditions of fairness for $p_{B}^{2}$ up to $N=5$. When playing only game $B(\gamma=0)$, the following symmetry in the stationary distribution can be deduced from Eq. (6):

$$
P_{i}^{s t,\left\{p_{B}^{1}, p_{B}^{2}, p_{B}^{3}\right\}}=P_{N-i}^{s t,\left\{1-p_{B}^{3}, 1-p_{B}^{2}, 1-p_{B}^{1}\right\}} .
$$

This property implies that $p_{w i n}^{A+B}$ is unaltered by the parameter transformation: $\left\{p_{B}^{1}, p_{B}^{2}, p_{B}^{3}\right\} \rightarrow\left\{1-p_{B}^{3}\right.$, $\left.1-p_{B}^{2}, 1-p_{B}^{1}\right\}$. It also means that for the parameter set $\left\{p_{B}^{1}, p_{B}^{2}=\frac{1}{2}, 1-p_{B}^{1}\right\}$, the stationary probability 
Table 1

Condition on $p_{B}^{2}$ in order that game $B$ is fair for $N=2, \ldots, 5$

\begin{tabular}{ll}
\hline$N$ & $p_{B}^{2}$ \\
\hline 2 & $\frac{\frac{p_{B}^{1}-1}{p_{B}^{1}-p_{B}^{3}-1}}{3}$ \\
& $\frac{\left(p_{B}^{1}-1\right)\left(p_{B}^{3}+1\right)+\sqrt{\left(p_{B}^{1}-2\right)\left(p_{B}^{1}-1\right) p_{B}^{3}\left(p_{B}^{3}+1\right)}}{\left(p_{B}^{1}+p_{B}^{3}-1\right)}$ \\
5 & $\frac{\left(p_{B}^{1}-1\right)^{2}\left(p_{B}^{3}+1\right)}{1+p_{B}^{3}+\left(p_{B}^{1}-2\right)\left(p_{B}^{1}+p_{B}^{1} p_{B}^{3}-\left(p_{B}^{3}\right)^{2}\right)}$ \\
& {$\left[1-\frac{p_{B}^{3}}{p_{B}^{1}-1} \sqrt{\frac{5+2 p_{B}^{1}\left(p_{B}^{1}-3\right)}{1+2 p_{B}^{3}\left(1+p_{B}^{3}\right)}}\right]^{-1}$} \\
\hline
\end{tabular}
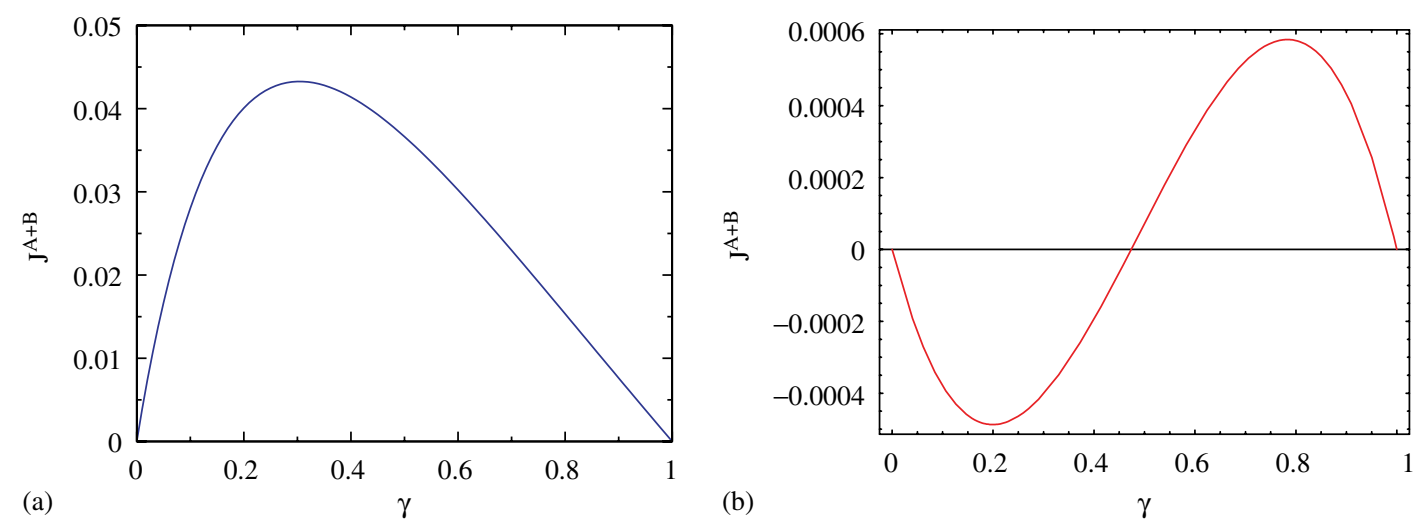

Fig. 2. (a) Plot of the current vs. the mixing probability $\gamma$ between games $A$ and $B$ for $N=4$ with probabilities $p^{A}=\frac{1}{2}, p_{B}^{1}=0.79$, $p_{B}^{2}=0.65$ and $p_{B}^{3}=0.15$. (b) Plot of the current vs. the mixing probability $\gamma$ between games $A$ and $B$ for $N=3$ with probabilities $p^{A}=\frac{1}{2}$, $p_{B}^{1}=0.686, p_{B}^{2}=0.423$ and $p_{B}^{3}=0.8$.

distribution is symmetric over the states, i.e., $P_{i}^{s t}=P_{N-i}^{s t}$. Therefore, when combining this with game $A$, i.e., alternating two games with symmetric probability distributions, always yields a fair game, independent of the values of $\gamma, N$ and $p_{B}^{1}$. To see the Parrondo effect, we need another, non-trivial, parameter set which yields a fair game $B$. For example, for $N=4$ we obtain a fair game $B$ when $p_{B}^{1}=0.79, p_{B}^{2}=0.65$ and $p_{B}^{3}=0.15$. The stochastic combination with game $A$ reproduces the desired Parrondo effect, see Fig. 2a.

\subsection{Results}

\subsubsection{Two players}

For $N=2$ players, there are three different states. Fig. 3a shows the regions in parameter space $\left\{\gamma, p_{B}^{1}, p_{B}^{3}\right\}$ where the mixing $(0<\gamma<1)$ between games $A$ and $B$ results in a fair, winning or losing game. Note that $p_{B}^{2}$ is fixed by the condition to have a fair game $B$, see Table 1 . Besides the case $p_{B}^{1}=1-p_{B}^{3}$, valid for any number of players, also $p_{B}^{1}=p_{B}^{3}$ results in a fair game for $N=2$, independent of the alternation probability $\gamma$. From Eq. (6), one can deduce that $p_{B}^{1}=p_{B}^{3}$ and $p_{B}^{1}=1-p_{B}^{3}$ imply a symmetric distribution $P_{i}^{s t}$ over the states, i.e., $P_{0}^{s t}=P_{2}^{s t}$. As mentioned before, this property prohibits any net current in the system. For all other values of $p_{B}^{1}$ and $p_{B}^{3}$ the Parrondo effect appears, that is, game $A+B$ is either a winning or a losing game, cf. Fig. 3a. 

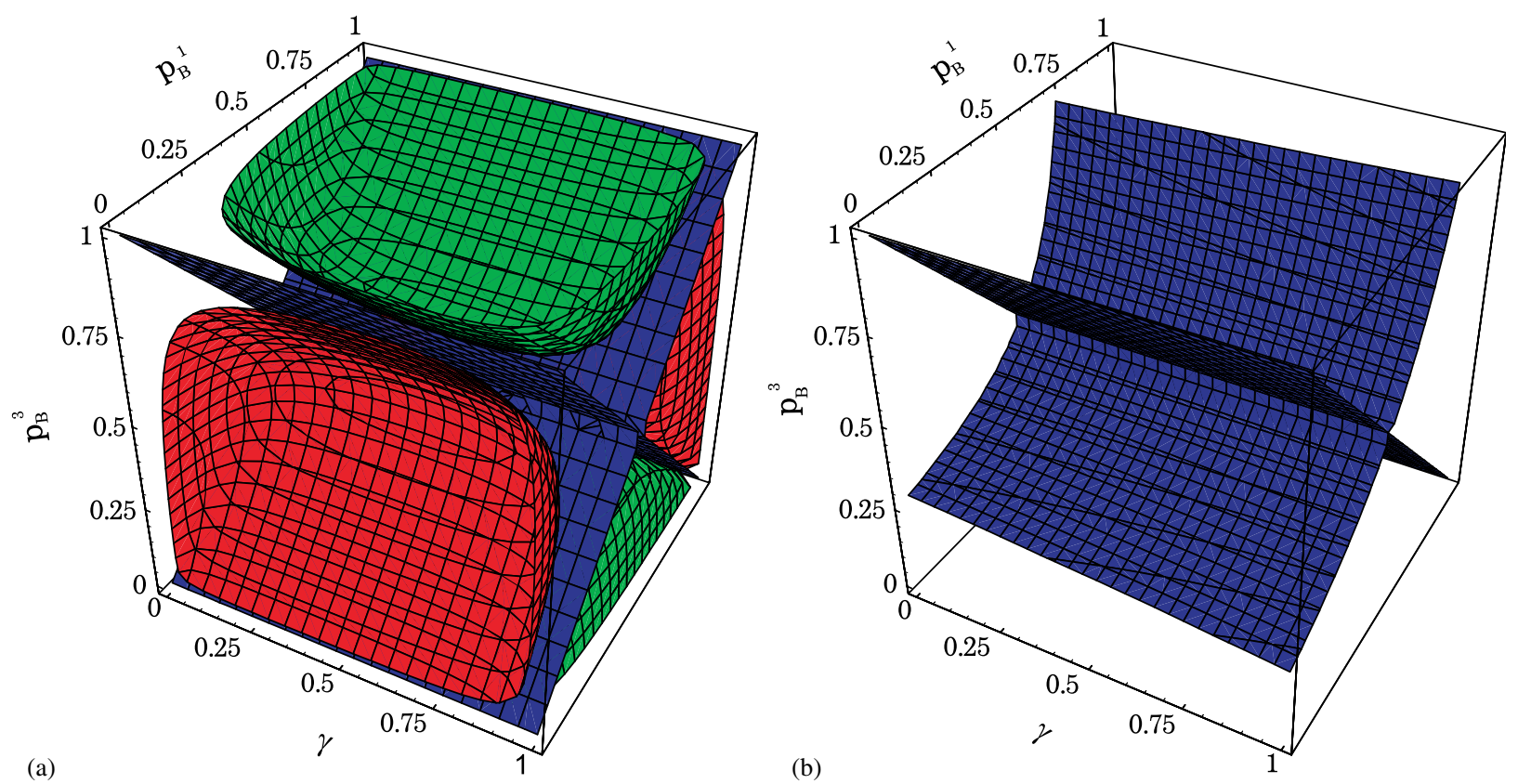

Fig. 3. (a) $N=2$. The regions in parameter space for which $p_{\text {win }}^{A+B}=0.5,0.499$ and 0.501 , indicating the regions where $A+B$ is fair (blue), losing (red) and winning (green), respectively. The blue diagonal planes show the situations $p_{B}^{1}=1-p_{B}^{3}$ and $p_{B}^{1}=p_{B}^{3}$, for which $A+B$ is fair, independent of $\gamma$. (b) $N=3$. The regions in parameter space for which the mixing $(0<\gamma<1)$ between game $A$ and $B$ results in a fair game. Besides the trivial diagonal plane, there is a curved plane - not uniform in $\gamma$ - for which $J^{A+B}=0$.

\subsubsection{Three players}

Fig. 3b shows for $N=3$ the surfaces in parameter space $\left\{\gamma, p_{B}^{1}, p_{B}^{3}\right\}$ where $A+B$ is a fair game. Besides the plane $p_{B}^{1}=1-p_{B}^{3}$, there is a second, curved surface with values of $\gamma$ different from 0 and 1 which results in $J^{A+B}=0$. This curved surface is not uniform in $\gamma$ and is therefore the collection of points of flux reversal between a winning and losing game $A+B$. This implies that, depending on the value of $\gamma$ we can either have a winning game or a losing game by alternating between two fair games. For example, in Fig. $2 \mathrm{~b}$ we have plotted the current $J^{A+B}$ vs. $\gamma$ for the set of probabilities $p^{A}=\frac{1}{2}, p_{B}^{1}=0.686, p_{B}^{2}=0.423$ and $p_{B}^{3}=0.8$. For low values of $\gamma$ the resulting game is a losing game, whereas for high values of $\gamma$ the game turns to be a winning game, cf. Fig. 2b. In both regions there exists an optimal value for $\gamma$ giving a maximum current. We can provide a qualitative picture that may help understanding the mechanism by which the current inversion phenomenon takes place.

When playing exclusively game $B(\gamma=0)$, the stationary distribution $P_{i}^{s t}$ is not homogeneous. This is reflected by the fact that the central states $\left\{\sigma_{1}, \sigma_{2}\right\}$ have a higher occupancy probability $\left(P_{i}^{s t}\right)$ than the boundary states $\left\{\sigma_{0}, \sigma_{3}\right\}$. On the other hand, if we look to the winning probability, it is higher in the latter set of states rather than in the former one $\left(p_{B}^{1}, p_{B}^{3}>p_{B}^{2}\right)$.

Indeed, the central states can be labelled as losing states, as when combining game $B$ with game $A$ for any $0 \leqslant \gamma<1$, the average losing probability $p_{i}^{l}=\gamma\left(1-p^{A}\right)+(1-\gamma)\left(1-p_{i}^{B}\right)<\frac{1}{2}$, i.e., it is more likely on average for a player to lose money rather than to win when being in one of these states. On the other hand, for the boundary states the contrary is true: it is more likely to win money rather than to lose for any $0 \leqslant \gamma<1$, so we can refer to them as winning sites, i.e., $p_{i}^{w}=\gamma p^{A}+(1-\gamma) p_{i}^{B}>\frac{1}{2}$.

When combining game $B$ with $A$, the resulting game will be fair, losing or winning depending on the net balance between the occupancy probabilities and the average winning probability on each set of central and boundary states. For low $\gamma$ values (playing game $B$ more often), the high occupancy probability of $\left\{\sigma_{1}, \sigma_{2}\right\}$ is the dominant part, and due to the low winning probability on these sites the resulting game is a losing game. On the contrary, for higher $\gamma$ values (playing game $A$ more often), the winning probability on the boundary sites $\left\{\sigma_{0}, \sigma_{3}\right\}$ is high enough to compensate their low occupancy, resulting in a winning game. 


\subsection{3. $N$ players}

For a general number of players, we have not been able to find the analytical expressions for a fair game $B$. Nevertheless, we will show numerically that the results for $N=3$ are representative for any $N$. This is illustrated by Fig. 4 , where the parameter space $\left\{p_{B}^{2}, p_{B}^{3}\right\}$ giving a fair game $B$ is shown, corresponding to a fixed $p_{B}^{1}=0.4$ and different values of $N$. As shown, the different curves seem to converge to a limiting curve as $N$ increases. Note that all curves intersect at the trivial point $\left\{p_{B}^{1}=0.4, p_{B}^{2}=0.5, p_{B}^{3}=0.6\right\}$.

We can also obtain the parameter space where the current inversion takes place, for different values of $N$. For clarity reasons we show in Fig. 5 only a vertical slice corresponding to a fixed $\gamma=0.4$, and different values of $N$. Again, the regions for which a flux inversion exists, does not seem to depend much on $N$. The only exception is $N=4$, for which the curve bends in the other direction. This is a consequence of the fact that for

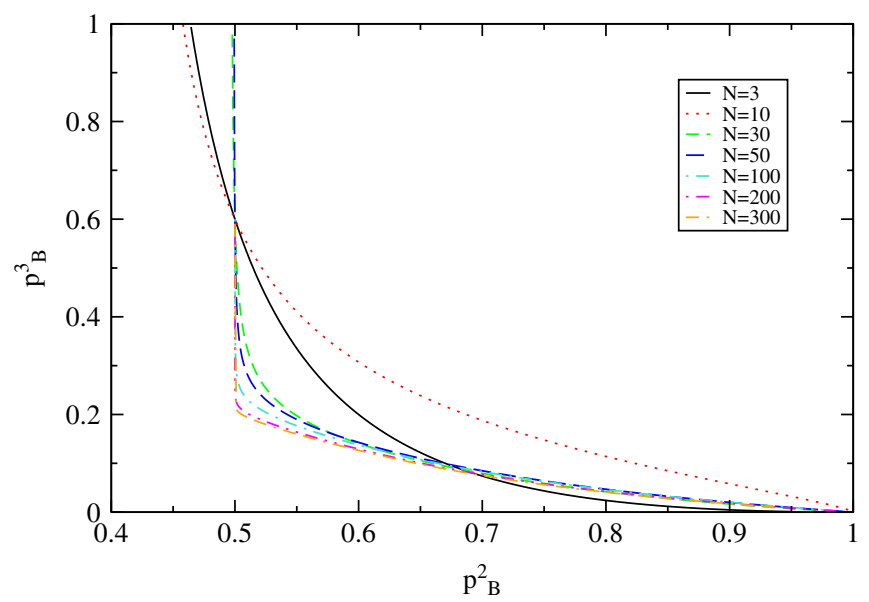

Fig. 4. Plot of the parameter space $\left\{p_{B}^{2}, p_{B}^{3}\right\}$ for a fixed $p_{B}^{1}=0.4$ that gives a fair game $B$ for different values of $N=3,10,30,50,100,200$ and 300 . As it can be seen, the curves seem to converge to a limiting curve as $N$ increases.

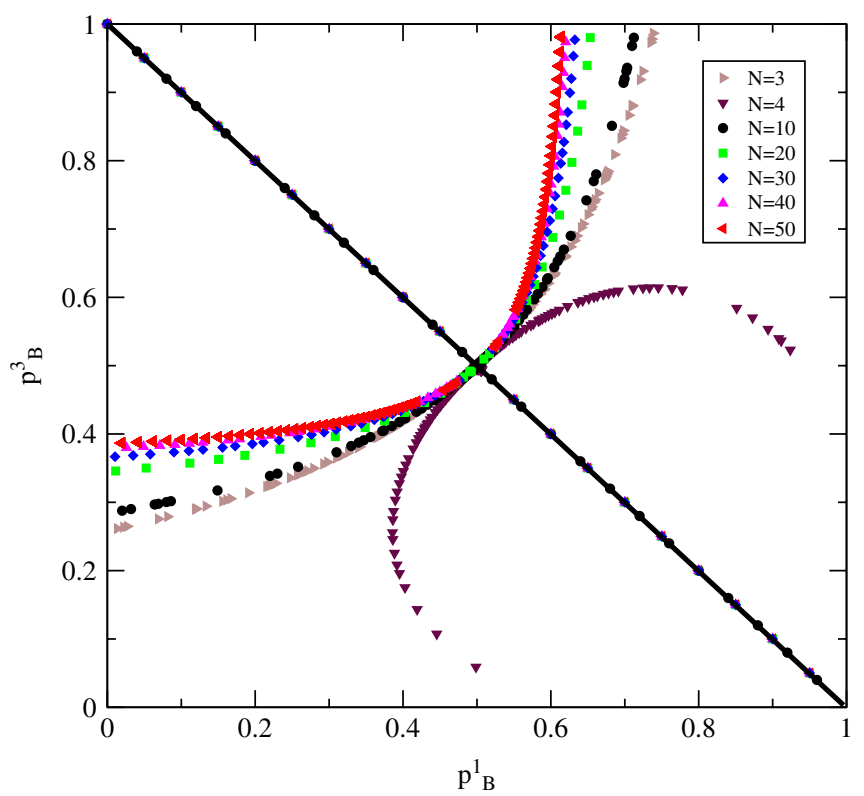

Fig. 5. Plot of the points in parameter space $\left\{p_{B}^{1}, p_{B}^{3}\right\}$ where (for $\gamma=0.4$ fixed) $A+B$ is a fair game. Results for different values of the total number of players $N=3,4,10,20,30,40$ and 50 are shown. The diagonal line shows the common plane $p_{B}^{1}=1-p_{B}^{3}$, that corresponds to a fair game $B$ for any number of players $N$. 
$N=4$ there exists only one state (namely $\sigma_{2}$ ) where the probability $p_{B}^{2}$ is used. This is confirmed by our findings when we modify the definition of game $B$ such that there is for any $N$ only one state where $p_{B}^{2}$ is used. The fact that all curves of inversion points are symmetric upon reflection about the plane $p_{B}^{1}=1-p_{B}^{3}$ is a consequence of the property of Eq. (8).

\section{Parrondo's games and the current inversion}

As stated previously and shown in Ref. [10], the effect of a current inversion when varying the mixing probability $\gamma$ is not possible when combining the original game $B$ with a state independent type game $A$. One way of understanding the reason is by means of the relation that has been established recently $[6,7,11]$ between the Brownian ratchet and Parrondo's games. A fair or unfair paradoxical game corresponds to a periodic or tilted potential respectively in the model of a Brownian ratchet.

As an illustration, we have depicted the potential corresponding to the original game $B$ in Fig. 6a. If we now combine game $B$ with $A$-which would have an associated flat potential - with a certain probability $\gamma$, the potential obtained is no longer periodic, i.e., it is tilted to the right in agreement with the direction of the flux, see Fig. 6b. Therefore, the question now reduces to explain why there is no current inversion in the flashing ratchet model when varying the rate of alternation between the potentials.

In the flashing ratchet model, the appearance of a flux when alternating between a flat and an asymmetric potential is due to a rectification process: if we consider a bunch of Brownian particles subjected to a ratchet potential, they will tend to remain in the potential well for a sufficiently small temperature - see Fig. 7a. When the potential is switched off the particles start diffusing, and if we wait for long enough, it is more likely that a small fraction of particles will reach the vicinity of the potential well located to the right, rather than the one on the left, due to the geometry of the potential. Switching on and off the potential many times, generates a net flux to the right. Hence, whatever the rate of alternation between these two potentials, it will always be more likely for a particle to move rightwards rather leftwards. Therefore, it will be impossible to obtain a current reversal by means of varying only the flip rate of the potentials.

\section{Conclusions}

We have presented a new type of collective Parrondo games. These games present, besides the Parrondo effect, a current inversion when varying the alternation probability $\gamma$ between the two games $A$ and $B$. The novelty introduced in this work relies on the fact that the current inversion appears from the combination of a collective game - i.e., game $B$ - and a totally unbiased, state independent, game $A$. Analytical expressions for

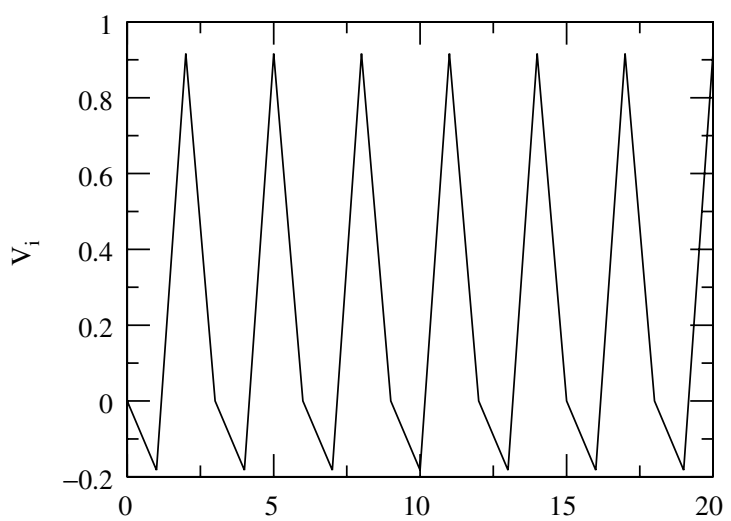

(a)

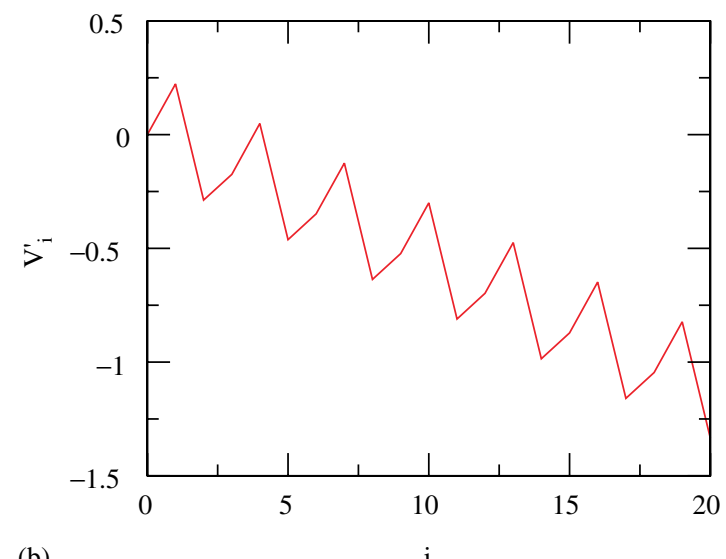

(b)

Fig. 6. (a) Plot of the potential related to the original Parrondo game $B$ obtained with the relation described in Ref. [6]. (b) Effective potential that we obtain when alternating between the original Parrondo games $A$ and $B$ with probability $\gamma=\frac{1}{2}$. 
ON

(a)

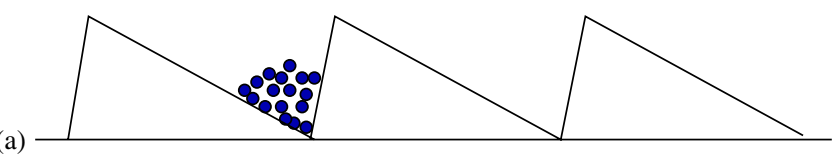

OFF

(b)

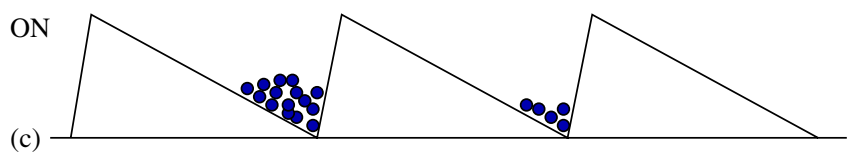

Fig. 7. Different stages of the mechanism of rectification when switching a ratchet potential on and off.

the games have been obtained for a finite number of players using discrete-time Markov chain techniques. We have also explained qualitatively the reason of this current inversion.

It remains as an open question the possible implications of these findings in the field of the Brownian ratchet, as well as the possibility of finding a physical model equivalent to this collective game.

\section{Acknowledgements}

The authors thank C. Van den Broeck for very helpful discussions. P.A. and R.T. acknowledge financial support from the Ministerio de Educación y Ciencia (Spain), FEDER projects FIS2004-5073, FIS2004-953. PA is supported from the local government of the Balearic Islands.

\section{References}

[1] G.P. Harmer, D. Abbott, Losing strategies can win by Parrondo's paradox, Nature 402 (1999) 864.

[2] G.P. Harmer, D. Abbott, A review of Parrondo's paradox, Fluctuations Noise Lett. 2 (2002) R71-R107.

[3] J.M.R. Parrondo, L. Dinís, Brownian motion and gambling: from ratchets to paradoxical games, Contemp. Phys. 45 (2004) $147-157$.

[4] J.M.R. Parrondo, How to cheat a bad mathematician, EEC HC\&M Network on Complexity and Chaos (\#ERBCHRX-CT 940 546), Torino, Italy, 1996, unpublished.

[5] P. Reimann, Brownian motors: noisy transport far from equilibrium, Phys. Rep. 361 (2002) 57-265.

[6] R. Toral, P. Amengual, S. Mangioni, Parrondo's games as a discrete ratchet, Physica A 327 (2003) $105-110$.

[7] R. Toral, P. Amengual, S. Mangioni, A Fokker-Planck description for Parrondo's games, in: Proceedings of SPIE Noise in Complex Systems and Stochastic Dynamics, vol. 5114, 2003, pp. 309-317.

[8] R. Toral, Cooperative Parrondo's games, Fluctuations Noise Lett. 1 (2001) L7-L12.

[9] R. Toral, Capital redistribution brings wealth by Parrondo's paradox, Fluctuations Noise Lett. 2 (2002) L305-L311.

[10] H. Moraal, Counterintuitive behaviour in games based on spin models, J. Phys. A 33 (2000) L203-L206.

[11] P. Amengual, R. Toral, Exact ratchet description of Parrondo's games with self-transitions, Noise in complex systems and stochastic dynamics II, Proc. SPIE 5471 (2004) 407-415. 